VOL. $72(2005) \quad[299-315]$

\title{
PROPER 1-BALL CONTRACTIVE RETRACTIONS IN BANACH SPACES OF MEASURABLE FUNCTIONS
}

\author{
D. Caponetti, A. Trombetta and G. Trombetta
}

\begin{abstract}
In this paper we consider the Wośko problem of evaluating, in an infinite-dimensional Banach space $X$, the infimum of all $k \geqslant 1$ for which there exists a $k$-ball contractive retraction of the unit ball onto its boundary. We prove that in some classical Banach spaces the best possible value 1 is attained. Moreover we give estimates of the lower $\mathrm{H}$-measure of noncompactness of the retractions we construct.
\end{abstract}

\section{INTRODUCTION}

Let $X$ be an infinite-dimensional Banach space with unit closed ball $B(X)$ and unit sphere $S(X)$. It is well known that, in this setting, there is a retraction of $B(X)$ onto $S(X)$, that is, a continuous mapping $R: B(X) \rightarrow S(X)$ with $R x=x$ for all $x \in S(X)$. In [4] Benyamini and Sternfeld, following Nowak ([13]), proved that such a retraction can be chosen among Lipschitz mappings. The problem of evaluating the infimum $k_{0}(X)$ of the Lipschitz constants of such retractions is of considerable interest in the literature. A general result states that in any Banach space $X, 3 \leqslant k_{0}(X) \leqslant k_{0}$ (see $\left.[8,10]\right)$, where $k_{0}$ is a universal constant. In special spaces more precise estimates have been obtained by means of constructions which depend on each space. We refer the reader to $[\mathbf{9}, \mathbf{1 0}]$ for a collection of results on this problem and related ones.

A similar problem can be considered by replacing Lipschitz retractions by $k$-ball contractive retractions. Let us recall that for a bounded $A \subset X$, the Hausdorff measure (briefly $H$-measure) of noncompactness $\gamma(A)$ is the infimum of all $\varepsilon>0$ such that $A$ has a finite $\varepsilon$-net in $X$. The following properties of $\gamma$ hold, for bounded $A, B \subset X$ :

$\gamma(A)=0$ if and only if $A$ is precompact;

$\gamma(\overline{c o} A)=\gamma(A)$ where $\overline{c o} A$ denotes the closed convex hull of $A$;

$\gamma(A \cup B)=\max \{\gamma(A), \gamma(B)\}$;

$\gamma(A+B) \leqslant \gamma(A)+\gamma(B)$

$\dot{\gamma}(\lambda A)=|\lambda| \gamma(A)$, for all $\lambda \in \mathbb{R}$.

Received 5th May, 2005

Copyright Clearance Centre, Inc. Serial-fee code: 0004-9727/05 \$A2.00+0.00. 
A continuous mapping $T: \operatorname{dom}(T) \subset X \rightarrow X$ is called $k$-ball contractive if there is $k \geqslant 0$ such that $\gamma(T A) \leqslant k \gamma(A)$ for each bounded $A \subset \operatorname{dom}(T)$.

In [20] Wośko has proved that in the space $X=C([0,1])$ for any $\varepsilon>0$ there exists a $(1+\varepsilon)$-ball contractive retraction of $B(X)$ onto $S(X)$. Moreover he has posed the question of estimating the characteristic:

$$
W(X)=\inf \{k \geqslant 1: \text { there is a } k \text {-ball contractive retraction } R: B(X) \rightarrow S(X)\}
$$

for special classical Banach spaces, and also the question whether or not there is a Banach space in which $W(X)$ is a minimum. As Wośko has pointed out a 1-ball contractive retraction cannot be a Lipschitz mapping. In [19] it was shown that $W(X) \leqslant 6$ for any Banach space, reaching the value 4 and 3 depending on the geometry of the space $X$. Results in other Banach spaces can be found in $[6,12,16,17]$. Recently, in $[1$, Theorem 4] it has been proved that if the Banach space $X$ has a monotone norm, then for any $\varepsilon>0$ there exists a $(1+\varepsilon)$-ball contractive retraction of $B(X)$ onto $S(X)$. For a continuous mapping $T: \operatorname{dom}(T) \subset X \rightarrow X$ we also consider the following quantitative characteristic which is of interest in nonlinear analysis:

$$
\omega(T)=\sup \{k \geqslant 0: \gamma(T A) \geqslant k \gamma(A) \text { for every bounded } A \subset \operatorname{dom}(T)\},
$$

called the lower H-measure of noncompactness of $T$. This characteristic is closed related to properness. In fact, from $\omega(T)>0$ it follows that $T$ is a proper mapping, that is, $T^{-1} K$ is compact for each compact subset $K$ of $X$.

Aim of this paper is to estimate $W(X)$ in some classical Banach spaces of real valued measurable functions on $[0,1]$ and also to give estimates of the lower $\mathrm{H}$-measure of noncompactness of the retractions we construct. In Section 3 we consider special Banach spaces in which, by means of a suitable compact mapping $P_{X}: B(X) \rightarrow X$, we give an explicit formula of a $k$-ball contractive retraction with positive lower H-measure of noncompactness. In the sections which follow we give examples of Banach spaces $X$ in which $W(X)=1$. In Orlicz (Section 4) and Lorentz spaces (Section 5) we obtain that the value $W(X)=1$ is actually a minimum. Moreover in Lebesgue and Lorentz spaces we show that a 1-ball contractive retraction $R$ can be chosen in such a way that $\omega(R)=1$. As a consequence in the Lebesgue and Lorentz spaces we have the existence of 1-ball contractive fixed point free mappings $F: B(X) \rightarrow B(X)$ with $\omega(F)=1$.

\section{Preliminaries.}

Let $\Sigma$ be the $\sigma$-algebra of all Lebesgue measurable subsets of $[0,1]$ equipped with the Lebesgue measure $\mu$, and write almost everywhere for $\mu$-almost everywhere. Let $\mathcal{M}_{0}:=\mathcal{M}_{0}([0,1], \Sigma, \mu)$ denote the space of all classes of Lebesgue measurable functions $f:[0,1] \rightarrow \mathbb{R}$ and $\mathcal{M}_{0}^{+}$its positive cone. We recall the definition of Banach function space, we refer to the book of Bennett-Sharpley [3] for the main results of this theory. 
DEFINITION 2.1. A mapping $\rho: \mathcal{M}_{0}^{+} \rightarrow[0, \infty]$ is called a Banach function norm if, for all $f, g, f_{n}(n=1,2, \ldots)$ in $\mathcal{M}_{0}^{+}$, for all constants $\lambda \geqslant 0$ and for all $E \in \Sigma$, the following properties hold:

$$
\begin{aligned}
& \rho(f)=0 \text { if and only if } f=0 \text { almost everywhere in }[0,1] ; \\
& \rho(\lambda f)=\lambda \rho(f) ; \\
& \rho(f+g) \leqslant \rho(f)+\rho(g) ; \\
& g \leqslant f \text { almost everywhere } \Rightarrow \rho(g) \leqslant \rho(f) ; \\
& f_{n} \uparrow f \text { almost everywhere } \Rightarrow \rho\left(f_{n}\right) \uparrow \rho(f) ; \\
& \rho\left(\chi_{[0,1]}\right)<\infty ; \\
& \int_{E} f(t) d t \leqslant C_{E} \rho(f), \text { for some constant } 0<C_{E}<\infty \text { independent of } f .
\end{aligned}
$$

DEFINITION 2.2. If $\rho$ is a Banach function norm, the Banach space

$$
Y=\left\{f \in \mathcal{M}_{0}: \rho(|f|)<\infty\right\}
$$

is a Banach function space, endowed with the norm $\|f\|=\rho(|f|)$.

Throughout this section $Y$ is a Banach function space.

DEFINITION 2.3. A function $f \in Y$ is said to have absolutely continuous norm if for every $\varepsilon>0$ there is $\delta>0$ such that $\left\|f \chi_{D}\right\|<\varepsilon$ for every $D \in \Sigma$ with $\mu(D)<\delta$.

Note that, as the underlying space $[0,1]$ has finite measure, by virtue of $[18$, Lemma 3.3.2], the above definition is equivalent to [3, Definition 3.1]. We set

$$
Y^{0}=\{f \in Y: f \text { has absolutely continuous norm }\} .
$$

If $Y^{0}=Y$, then the space $Y$ is said to have absolutely continuous norm. We denote by $W$ the set of all simple functions of $\mathcal{M}_{\mathbf{0}}$. We recall that $W$ is a subset of $Y$ and we denote by $\bar{W}^{\|\cdot\|}$ the closure of $W$ in $Y$.

The next lemma collects some results we need (see [3, Theorems 3.8, 3.11 and 3.13]).

LEMMA 2.4. The following statements hold:

(i) The space $Y^{0}$ is an order ideal of $Y$, that is, it is a closed linear subspace of $Y$ with the property:

$$
f \in Y^{0} \text { and }|g| \leqslant|f| \text { almost everywhere } \Rightarrow g \in Y^{0} .
$$

(ii) The subspace $\bar{W}^{\|\cdot\|}$ is an order ideal of $Y$ and $Y^{0} \subset \bar{W}^{\|\cdot\|} \subset Y$.

(iii) The subspaces $Y^{0}$ and $\bar{W}^{\|\cdot\|}$ coincide if and only if the characteristic function $\chi_{[0,1]}$ has absolutely continuous norm. In particular, $Y^{0}=\bar{W}^{\|\cdot\|}=Y$ whenever $Y$ has absolutely continuous norm.

We recall the following useful characterisation of convergent sequences in $Y^{0}$. 
Lemma 2.5. ([2, p. 41]) $A$ sequence $\left\{f_{n}\right\}$ converges to $f$ in $Y^{0}$ if and only if $\left\{f_{n}\right\}$ converges to $f$ in measure and the family $\left\{f_{n}: n \in N\right\}$ has uniformly absolutely continuous norm, that is, for every $\varepsilon>0$ there is $\delta>0$ such that $\sup _{n}\left\|f_{n} \chi_{D}\right\|<\varepsilon$ for every $D \in \Sigma$ with $\mu(D)<\delta$.

Let $C([0,1])$ denote the Banach space of all real and continuous functions on $[0,1]$ endowed with the sup norm $\|\cdot\|_{\infty}$. By a standard argument (see for example [15, Theorem 3.14]) it can be shown the following lemma.

LEMMA 2.6. Assume $Y^{0}=\bar{W}^{\|\cdot\|}$, then $C([0,1])$ is dense in $Y^{0}$.

\section{Proper $k$-Ball CONTRACtive Retractions: abstract Results.}

Let $X$ denote the Banach space of all functions of absolutely continuous norm of a Banach function space $Y$. We still denote by $W$ the subset of $Y$ of all simple functions. For $f \in X$ and $a \in[1,2]$, we set

$$
f_{a}(t)=\left\{\begin{array}{lll}
f(a t) & \text { if } & t \in\left[0, \frac{1}{a}\right] \\
0 & \text { if } & t \in\left(\frac{1}{a}, 1\right]
\end{array}\right.
$$

Throughout this section we assume that the Banach space $X$ satisfies the following properties:

(P1) $X=\bar{W}^{\|\cdot\|}$;

(P2) there is a continuous decreasing function $\alpha:[1,2] \rightarrow \mathbb{R}$ with $\alpha(1)=1$ and $\alpha(2)>0$ such that

$$
\alpha(a)\|f\| \leqslant\left\|f_{a}\right\| \leqslant\|f\|,
$$

for every $f \in X$ and $a \in[1,2]$. Then it is easy to check that $f_{\mathrm{a}} \in X$.

Now for any continuous function $g \in X$ we set $A_{g}=\left\{g_{a}: a \in[1,2]\right\}$. We need the following two lemmas, the proofs of which are straightforward.

LEMMA 3.1. Let $g \in X$ be continuous. Then the set $A_{g}$ is compact.

PRoOf: Let $g \in X$ be continuous. For any $a \in[1,2]$, we have $\left|g_{a}\right| \leqslant\|g\|_{\infty} \chi[0,1]$ then (1) implies

$$
\left\|g_{a}\right\| \leqslant\|g\|_{\infty}\|\chi[0,1]\| \text {. }
$$

From the last inequality it follows that $A_{g}$ has uniformly absolutely continuous norm. Let now $\left\{g_{a_{n}}\right\}$ be a sequence of elements of $A_{g}$. Choose a subsequence $\left\{a_{n_{k}}\right\}$ of $\left\{a_{n}\right\}$ which is convergent, say to $a$. It is easy to check that $g_{a_{n_{k}}} \rightarrow g_{a}$ almost everywhere in $[0,1]$, so that $g_{a_{n_{k}}} \rightarrow g_{a}$ in measure. By Lemma 2.5 , the thesis follows.

LEMmA 3.2. Let $g \in X$ be continuous and $a_{n} \rightarrow a\left(a_{n} \in[1,2]\right)$. Then $\left\|g_{a_{n}}-g_{a}\right\| \rightarrow 0$. 
Proof: Let $g \in X$ be continuous and $a_{n} \rightarrow a\left(a_{n} \in[1,2]\right)$. Given $\varepsilon>0$, as $A_{g}$ has uniformly absolutely continuous norm, there exists $\delta>0$ such that $\left\|g_{a} \chi_{D}\right\|<\varepsilon$ and $\left\|g_{a_{n}} \chi_{D}\right\|<\varepsilon$ for all $n \in N$ whenever $D \in \Sigma$ and $\mu(D)<\delta$. Find an index $\nu$ such that for all $n \geqslant \nu$ we have $1 / a_{n} \in(1 / a-\delta / 2,1 / a+\delta / 2)$ and $\left|g\left(a_{n} t\right)-g(a t)\right| \leqslant \varepsilon$ for all $t \in[0,1]$ with $t \leqslant 1 / a-\delta / 2$. Then $\sup _{[0,1 / a-\delta / 2]}\left|g_{a_{n}}(t)-g_{a}(t)\right| \leqslant \varepsilon$ and so

$$
\left\|\left(g_{a_{n}}-g_{a}\right) \chi_{[0,1 / a-\delta / 2]}\right\| \leqslant \varepsilon\left\|\chi_{[0,1]}\right\| .
$$

Hence for every $n \geqslant \nu$ we have

$$
\begin{aligned}
\left\|g_{a_{n}}-g_{a}\right\| & \leqslant\left\|\left(g_{a_{n}}-g_{a}\right) \chi_{[0,1 / a-\delta / 2]}\right\|+\left\|\left(g_{a_{n}}-g_{a}\right) \chi_{[1 / a-\delta / 2,1 / a+\delta / 2]}\right\| \\
& \leqslant \varepsilon\left\|\chi_{[0,1]}\right\|+2 \varepsilon
\end{aligned}
$$

and the thesis follows.

REMARK 3.3. If $a_{n} \rightarrow a\left(a_{n} \in[1,2]\right)$ by the same argument of Lemma 3.2 we have

$$
\left\|\chi_{\left(1 / a_{n}, 1\right]}-\chi_{(1 / a, 1]}\right\| \rightarrow 0 .
$$

We now define a mapping $Q: B(X) \rightarrow B(X)$ and establish the properties of $Q$ we need. The explicit formula of a retraction $R$, of which we can estimate the H-measure of noncompactness (that is, the infimum of all $k \geqslant 1$ for which $R$ is a $k$-ball contractive retraction) and the lower $\mathrm{H}$-measure of noncompactness, will depend on a suitable compact mapping $P_{X}: B(X) \rightarrow X$ satisfying the hypotheses of the subsequent Theorem 3.6. To define $Q: B(X) \rightarrow B(X)$ we set

$$
(Q f)(t)=f_{2 /(1+\|f\|)}(t), \text { for all } t \in[0,1] .
$$

We clearly have $Q f=f$ for all $f \in S(X)$.

PROPOSITION 3.4. The mapping $Q$ is continuous.

Proof: Let $\left\{f_{n}\right\}$ be a sequence of elements of $B(X)$ such that $\left\|f_{n}-f\right\| \rightarrow 0$. Let $\varepsilon>0$. By Lemma 2.6 there is a continuous $g \in B(X)$ such that $\|f-g\| \leqslant \varepsilon$. Choose and index $\nu$ such that for all $n \geqslant \nu$ we have $\left\|f-f_{n}\right\| \leqslant \varepsilon$, by Lemma 3.2 we may also assume $\left\|g_{2 /\left(1+\left\|f_{n}\right\|\right)}-g_{2 /(1+\|f\|)}\right\| \leqslant \varepsilon$. Using the last inequality and the right hand side of (2) we get, for all $n \geqslant \nu$

$$
\begin{aligned}
\left\|Q f_{n}-Q f\right\| \leqslant\left\|\left(f_{n}\right)_{2 /\left(1+\left\|f_{n}\right\|\right)}-f_{2 /\left(1+\left\|f_{n}\right\|\right)}\right\|+\left\|f_{2 /\left(1+\left\|f_{n}\right\|\right)}-g_{2 /\left(1+\left\|f_{n}\right\|\right)}\right\| \\
\quad+\left\|g_{2 /\left(1+\left\|f_{n}\right\|\right)}-g_{2 /(1+\|f\|)}\right\|+\left\|g_{2 /(1+\|f\|)}-f_{2 /(1+\|f\|)}\right\| \\
=\left\|\left(f_{n}-f\right)_{2 /\left(1+\left\|f_{n}\right\|\right)}\right\|+\left\|(f-g)_{2 /\left(1+\left\|f_{n}\right\|\right)}\right\| \\
\quad+\left\|g_{2 /\left(1+\left\|f_{n}\right\|\right)}-g_{2 /(1+\|f\|)}\right\|+\left\|(g-f)_{2 /(1+\|f\|)}\right\| \leqslant 4 \varepsilon
\end{aligned}
$$

which gives the thesis. 
Proposition 3.5. Let $A \subset B(X)$. Then

$$
\alpha(2) \gamma(A) \leqslant \gamma(Q A) \leqslant \gamma(A) .
$$

Proof: Let $A \subset B(X)$. We prove the right inequality. Let $\beta>\gamma(A)$. By Lemma 2.6, $C([0,1])$ is dense in $X$, thus there exists a $\beta$-net $\left\{\varphi_{1}, \ldots, \varphi_{p}\right\}$ for $A$ in $C([0,1])$. By Lemma 3.1 the set $\bigcup_{i=1}^{p} A_{\varphi_{i}}$ is compact, hence given $\delta>0$ we can choose a $\delta$-net $\left\{\psi_{1}, \ldots, \psi_{q}\right\}$ for $\bigcup_{i=1}^{p} A_{\varphi_{i}}$ in $X$. We now show that $\left\{\psi_{1}, \ldots, \psi_{q}\right\}$ is a $(\beta+\delta)$-net for $Q A$ in $X$.

Let $g \in Q A$ and let $f \in A$ be such that $Q f=g$. Fix $i \in\{1, \ldots, p\}$ such that $\left\|f-\varphi_{i}\right\| \leqslant \beta$. Since $\left(\varphi_{i}\right)_{2 /(1+\|f\|)} \in A_{\varphi_{i}}$ we can find $j \in\{1, \ldots, q\}$ such that

$$
\left\|\left(\varphi_{i}\right)_{2 /(1+\|f\|)}-\psi_{j}\right\| \leqslant \delta
$$

Then

$$
\begin{aligned}
\left\|Q f-\psi_{j}\right\| & \leqslant\left\|f_{2 /(1+\|f\|)}-\left(\varphi_{i}\right)_{2 /(1+\|f\|)}\right\|+\left\|\left(\varphi_{i}\right)_{2 /(1+\|f\|)}-\psi_{j}\right\| \\
& \leqslant\left\|f-\varphi_{i}\right\|+\delta \leqslant \beta+\delta .
\end{aligned}
$$

Therefore $\gamma(Q A) \leqslant \beta+\delta$, so $\gamma(Q A) \leqslant \gamma(A)$.

We now prove the left inequality. Let $\eta>\gamma(Q A)$. As $C([0,1])$ is dense in $X$, there exists an $\eta$-net $\left\{\lambda_{1}, \ldots, \lambda_{n}\right\}$ for $Q A$ in $C([0,1])$. For $i=1 \ldots, n$, set $\left(\lambda_{i}\right)^{b}(t)=\lambda_{i}(b t)$ for $t \in[0,1]$ and $b \in[1 / 2,1]$. Since each $\left(\lambda_{i}\right)^{b}$ is a continuous mapping, the set $\bigcup_{i=1}^{n}\left\{\left(\lambda_{i}\right)^{b}\right.$ : $b \in[1 / 2,1]\}$ is compact with respect to the $\|\cdot\|_{\infty}$ norm and hence is compact in $X$. Hence for any $\delta>0$ we can choose a $\delta$-net $\left\{\xi_{1}, \ldots, \xi_{m}\right\}$ for $\bigcup_{i=1}^{n}\left\{\left(\lambda_{i}\right)^{b}: b \in[1 / 2,1]\right\}$ in $X$. We now show that $\left\{\xi_{1}, \ldots, \xi_{m}\right\}$ is an $(\eta / \alpha(2)+\delta)$-net for $A$ in $X$.

Let $f \in A$. Fix $i \in\{1, \ldots, n\}$ such that $\left\|Q f-\lambda_{i}\right\| \leqslant \eta$. Since

$$
\left(\lambda_{i}\right)^{(1+\| f \mid 1) / 2} \in\left\{\left(\lambda_{i}\right)^{b}: b \in[1 / 2,1]\right\}
$$

we can find $j \in\{1, \ldots, m\}$ such that $\left\|\left(\lambda_{i}\right)^{(1+\|f\|) / 2}-\xi_{j}\right\| \leqslant \delta$. Then

$$
\begin{aligned}
\left\|f-\xi_{j}\right\| & \leqslant\left\|f-\left(\lambda_{i}\right)^{(1+\|f\|) / 2}\right\|+\left\|\left(\lambda_{i}\right)^{(1+\|f\|) / 2}-\xi_{j}\right\| \\
& \leqslant \frac{1}{\alpha(2)}\left\|f_{2 /(1+\|f\|)}-\left(\left(\lambda_{i}\right)^{(1+\|f\|) / 2}\right)_{2 /(1+\|f\|)}\right\|+\delta \\
& \leqslant \frac{1}{\alpha(2)}\left\|Q f-\lambda_{i}\right\|+\delta \leqslant \frac{\eta}{\alpha(2)}+\delta .
\end{aligned}
$$

Therefore $\gamma(A) \leqslant \eta / \alpha(2)+\delta$, so $\alpha(2) \gamma(A) \leqslant \gamma(Q A)$.

Theorem 3.6. Let $P_{X}: B(X) \rightarrow X$ be a compact mapping with $P_{X} f=0$ for all $f \in S(X)$, and

$$
\left\|Q f+P_{X} f\right\| \geqslant m
$$


for some $m \in(0,1]$ and all $f \in B(X)$. Then the mapping $R: B(X) \rightarrow S(X)$ defined by

$$
R f=\frac{Q f+P_{X} f}{\left\|Q f+P_{X} f\right\|}
$$

is a $(1 / m)$-ball contractive retraction. Moreover $\omega(R) \geqslant \alpha(2) / l$ whenever $\left\|Q f+P_{X} f\right\| \leqslant l$ for all $f \in B(X)$. In particular, if $\left\|Q f+P_{X} f\right\|=1$ for all $f \in B(X)$, the retraction $R$ is 1-ball contractive and $\omega(R) \geqslant \alpha(2)$.

Proof: Clearly the mapping $R$ defined in (5) is a retraction. Let $A \subset B(X)$. Since $P_{X}$ is compact, it follows from Proposition 3.5 that

$$
\alpha(2) \gamma(A) \leqslant \gamma\left(\left(Q+P_{X}\right) A\right) \leqslant \gamma(A) .
$$

Moreover by the definition of $R$ and by (4) we get

$$
R A \subset\left[0, \frac{1}{m}\right] \cdot\left(Q+P_{X}\right) A .
$$

Using the properties of $\gamma$, from (6) it follows $\gamma(R A) \leqslant(1 / m) \gamma(A)$. Similarly if $\| Q f$ $+P_{X} f \| \leqslant l$ for all $f \in B(X)$ we have

$$
\left(Q+P_{X}\right) A \subset[0, l] \cdot R A .
$$

Therefore $(\alpha(2) / l) \gamma(A) \leqslant \gamma(R A)$, and the proof is complete.

Observe that $\left\|Q f+P_{X} f\right\|=1$ for $f \in S(X)$, so in condition (4) we necessarily have $m \leqslant 1$.

REMARK 3.7. Whenever in a Banach space $X$ we find $\alpha(a)\|f\|=\left\|f_{a}\right\|$, for all $f \in B(X)$ (a stronger condition than (2)) we modify the mapping $Q$ defined in (3) by setting

$$
(Q f)(t)=\frac{1}{\alpha(2 /(1+\|f\|))} f_{2 /(1+\|f\|)}(t), \text { for all } t \in[0,1] .
$$

As no confusion can arise we keep denoting this mapping by $Q$. Then $\|Q f\|=\|f\|$ for all $f \in B(X)$. Clearly $Q$ is still a continuous mapping and, by slight modifications of the previous arguments and of Proposition 3.5, we get $\gamma(Q A)=\gamma(A)$. This allow us to obtain a better estimate of the lower H-measure of noncompactness of the retraction $R$ defined as in (5). In fact, under the same hypotheses of Theorem 3.6, we get $\omega(R) \geqslant 1 / l$.

COROLlary 3.8. The retraction $R$ defined in (5) is a proper mapping.

\section{The ORLicz SPACES $L_{\Phi}$.}

Let $\Phi:[0, \infty) \rightarrow[0, \infty)$ be a continuous strictly increasing Young's function. Assume that $\Phi$ satisfies the $\Delta_{2}$-condition, that is, there is $c \in[0, \infty)$ such that $\Phi(2 x) \leqslant c \Phi(x)(x \geqslant$ $0)$. For $f \in \mathcal{M}_{0}$ set

$$
M^{\Phi}(f)=\int_{[0,1]} \Phi(|f(t)|) d t
$$


Then

$$
\rho_{\Phi}(f)=\inf \left\{u>0: M^{\Phi}\left(\frac{f}{u}\right) \leqslant 1\right\}\left(f \in \mathcal{M}_{0}^{+}\right) .
$$

is a Banach function norm, and the Banach function space

$$
L_{\Phi}:=L_{\Phi}[0,1]=\left\{f \in \mathcal{M}_{0}: \rho_{\Phi}(|f|)<\infty\right\}
$$

is the Orlicz space generated by $\Phi$ endowed with the Luxemburg norm $\|f\|_{\Phi}=\rho_{\Phi}(|f|)$. The Orlicz space $L_{\Phi}$ is of absolutely continuous norm (see for example [14]). Then by Lemma 2.4 the space $L_{\Phi}$ satisfies property (P1). The following lemma proved in [12] shows that (P2) holds in $L_{\Phi}$.

LEMMA 4.1. ([12, Lemma 2.3]) Let $f \in L_{\Phi}$ and $a \in[1,2]$. Then

$$
\frac{1}{a}\|f\|_{\Phi} \leqslant\left\|f_{a}\right\|_{\Phi} \leqslant\|f\|_{\Phi}
$$

Let $Q: B\left(L_{\Phi}\right) \rightarrow B\left(L_{\Phi}\right)$ be defined as in (3) and define $P_{\Phi}: B\left(L_{\Phi}\right) \rightarrow L_{\Phi}$ by

$$
P_{\Phi} f= \begin{cases}\left.\Phi^{-1}\left(\frac{2}{1-\|f\|_{\Phi}}\left(1-M^{\Phi}(Q f)\right)\right) \chi\left(\left(1+\|f\|_{\Phi}\right) / 2\right), 1\right] & \text { if } f \in B\left(L_{\Phi}\right) \backslash S\left(L_{\Phi}\right) \\ 0 & \text { if } f \in S\left(L_{\Phi}\right) .\end{cases}
$$

Lemma 4.2. The mapping $P_{\Phi}$ is compact.

Proof: We prove that $P_{\Phi} B\left(L_{\Phi}\right)$ is relatively compact and $P_{\Phi}$ is continuous. Let $\left\{g_{n}\right\}$ be a sequence of elements of $P_{\Phi} B\left(L_{\Phi}\right)$ and $\left\{f_{n}\right\}$ be a sequence of elements of $B\left(L_{\Phi}\right)$ such that $P_{\Phi} f_{n}=g_{n}$, for all $n$. Since $0 \leqslant\left\|f_{n}\right\|_{\Phi} \leqslant 1$ and $0 \leqslant M^{\Phi}\left(Q f_{n}\right) \leqslant\left\|Q f_{n}\right\|_{\Phi} \leqslant 1$ for all $n$, we can choose subsequences $\left\{\left\|f_{n_{k}}\right\|_{\Phi}\right\},\left\{\left\|Q f_{n_{k}}\right\|_{\Phi}\right\}$ and $\left\{M^{\Phi}\left(Q f_{n_{k}}\right)\right\}$ which converge, say to $b, c$ and $c_{\Phi}$, respectively.

If $b=1$ then by Lemma $4.1,\left\|Q f_{n_{k}}\right\|_{\Phi} \rightarrow 1$ and consequently

$$
M^{\Phi}\left(Q f_{n_{k}}\right) \rightarrow 1
$$

Since $M^{\Phi}\left(P_{\Phi} f_{n_{k}}\right)=1-M^{\Phi}\left(Q f_{n_{k}}\right)$ we have $M^{\Phi}\left(P_{\Phi} f_{n_{k}}\right) \rightarrow 0$ and hence $\left\|P_{\Phi} f_{n_{k}}\right\|_{\Phi} \rightarrow 0$. This implies that $\left\{g_{n_{k}}\right\}$ converges in norm to the null function. Assume $b<1$ and write

$$
\begin{aligned}
& \left\|P_{\Phi} f_{n_{k}}-\Phi^{-1}\left(\frac{2}{1-b}\left(1-c_{\Phi}\right)\right) \chi_{((1+b) / 2,1)}\right\|_{\Phi} \\
& =\| \Phi^{-1}\left(\frac{2}{1-\left\|f_{n_{k}}\right\|_{\Phi}}\left(1-M^{\Phi}\left(Q f_{n_{k}}\right)\right)\right) \chi_{\left(\left(1+\left\|f_{n_{k}}\right\|_{\Phi}\right) / 2,1\right]} \\
& \quad-\Phi^{-1}\left(\frac{2}{1-b}\left(1-c_{\Phi}\right)\right) \chi_{((1+b) / 2,1]} \|_{\Phi}
\end{aligned}
$$

By Remark 3.3 we have

$$
\left\|\chi_{\left(\left(1+\left\|f_{n_{\xi}}\right\|_{\Phi}\right) / 2,1\right\}}-\chi_{((1+b) / 2,1]}\right\|_{\Phi} \rightarrow 0
$$


and by the continuity of $\Phi^{-1}$ we also have

$$
\Phi^{-1}\left(\frac{2}{1-\left\|f_{n_{k}}\right\|_{\Phi}}\left(1-M^{\Phi}\left(Q f_{n_{k}}\right)\right) \rightarrow \Phi^{-1}\left(\frac{2}{1-b}\left(1-c_{\Phi}\right)\right) .\right.
$$

Thus we get

$$
\left\|P_{\Phi} f_{n_{k}}-\Phi^{-1}\left(\frac{2}{1-b}\left(1-c_{\Phi}\right)\right) \chi_{((1+b / 2), 1]}\right\|_{\Phi} \rightarrow 0 \text {. }
$$

We have proved that $P_{\Phi} B\left(L_{\Phi}\right)$ is relatively compact.

Let now $\left\{f_{n}\right\}$ be a sequence of elements of $B\left(L_{\Phi}\right)$ such that $\left\|f_{n}-f\right\|_{\Phi} \rightarrow 0$, then, as the $\Delta_{2}$-condition holds, $M^{\Phi}\left(f_{n}\right) \rightarrow M^{\Phi}(f)$. An argument similar to that of the first part of the proof implies $\left\|P_{\Phi} f_{n}-P_{\Phi} f\right\|_{\Phi} \rightarrow 0$. The proof is complete.

LEMMA 4.3. Let $f \in B\left(L_{\Phi}\right)$, then

$$
\left\|Q f+P_{\Phi} f\right\|_{\Phi}=1
$$

Proof: Observe that, for any $u>0$ we have

$$
M^{\Phi}\left(\frac{Q f+P_{\Phi} f}{u}\right)=M^{\Phi}\left(\frac{Q f}{u}\right)+M^{\Phi}\left(\frac{P_{\Phi} f}{u}\right) .
$$

Now for $u=1$ we get

$$
\begin{aligned}
M^{\Phi}\left(Q f+P_{\Phi} f\right) & =\int_{\left(\left(1+\|f\|_{\Phi}\right) / 2,1\right]} \Phi\left(\Phi^{-1}\left(\frac{2}{1-\|f\|_{\Phi}}\left(1-M^{\Phi}(Q f)\right)\right)\right) d t+M^{\Phi}(Q f) \\
& =\int_{\left(\left(1+\|f\|_{\Phi} / 2\right), 1\right]} \frac{2}{1-\|f\|_{\Phi}}\left(1-M^{\Phi}(Q f)\right) d t+M^{\Phi}(Q f)=1
\end{aligned}
$$

It follows that $\left\|Q f+P_{\Phi} f\right\|_{\Phi} \leqslant 1$. On the other hand if $0<u<1$

$$
M^{\Phi}\left(\frac{Q f+P_{\Phi} f}{u}\right)>M^{\Phi}\left(Q f+P_{\Phi} f\right),
$$

consequently $\left\|Q f+P_{\Phi} f\right\|_{\Phi}=1$.

From Lemmas 4.1, 4.2 and 4.3 and Theorem 3.6 we obtain the following.

THEOREM 4.4. The mapping $R: B\left(L_{\Phi}\right) \rightarrow S\left(L_{\Phi}\right)$ defined by

$$
R f=Q f+P_{\Phi} f
$$

is a 1-ball contractive retraction and $\omega(R) \geqslant 1 / 2$.

Observe that, if $\Phi(t)=t^{p}$ where $1 \leqslant p<\infty$, then $L_{\Phi}$ is the Lebesgue space $L_{p}:=L_{p}[0,1]$, with the standard norm $\|\cdot\|_{p}$. But in this case an easy computation shows that $(1 / a)^{1 / p}\|f\|_{p}=\left\|f_{a}\right\|_{p}$. Hence, according to Remark 3.7, a stronger result on the characteristic $\omega(R)$ holds. Define $Q: B\left(L_{p}\right) \rightarrow B\left(L_{p}\right)$ (as in (7)) by

$$
(Q f)(t)=\left(\frac{2}{1+\|f\|_{p}}\right)^{1 / p} f_{2 /\left(1+\|f\|_{p}\right)}(t), \quad \text { for all } t \in[0,1] \text {. }
$$


Next define $P_{p}: B\left(L_{p}\right) \rightarrow L_{p}$ by

$$
P_{p} f= \begin{cases}\left(\frac{2}{1-\|f\|_{p}}\left(1-\|f\|_{p}^{p}\right)\right)^{1 / p} \chi_{\left(\left(1+\|f\|_{p}\right) / 2,1\right]} & \text { if } f \in B\left(L_{p}\right) \backslash S\left(L_{p}\right) \\ 0 & \text { if } f \in S\left(L_{p}\right) .\end{cases}
$$

Then the following theorem holds.

THEOREM 4.5. The mapping $R: B\left(L_{p}\right) \rightarrow S\left(L_{p}\right) \quad(1 \leqslant p<\infty)$ defined by

$$
R f=Q f+P_{p} f
$$

is a 1-ball contractive retraction and $\omega(R)=1$.

The results obtained in the Lebesgue spaces $L_{p}$ can be generalised to the weighted spaces. Let $\rho$ be a measurable weighting function. We consider the weighted Lebesgue space

$$
L_{p}(\rho):=L_{p}([0,1], \rho)(1 \leqslant p<\infty)
$$

which consists of all $f \in \mathcal{M}_{0}$ such that $\rho^{1 / p} f \in L_{p}$, endowed with the norm

$$
\|f\|_{L_{p}(\rho)}=\left(\int_{[0,1]} \rho(t)|f(t)|^{p} d t\right)^{1 / p}
$$

The space $L_{p}(\rho)$ has absolutely continuous norm.

We define a mapping $Q_{\rho}: B\left(L_{p}(\rho)\right) \rightarrow B\left(L_{p}(\rho)\right)$ by a slight modification of (7)

$$
\left(Q_{\rho} f\right)(t)=\left(\rho_{2 /\left(1+\|f\|_{L_{p}(\rho)}\right.}(t) / \rho(t)\right)^{1 / p}\left(\frac{2}{1+\|f\|_{L_{p}(\rho)}}\right)^{1 / p} f_{2 /\left(1+\|f\|_{L_{p}(\rho)}\right)}(t) \text { for all } t \in[0,1]
$$

and define $P_{\rho}: B\left(L_{p}(\rho)\right) \rightarrow L_{p}(\rho)$ by

$P_{\rho} f= \begin{cases}\left(\frac{2}{1-\|f\|_{L_{p}(\rho)}}\right)^{1 / p}\left(\frac{1-\|f\|_{L_{p}(\rho)}^{p}}{\rho(t)}\right)^{1 / p} \chi_{\left(\left(1+\|f\|_{p}\right) / 2,1\right]} & \text { if } f \in B\left(L_{p}(\rho)\right) \backslash S\left(L_{p}(\rho)\right) \\ 0 & \text { if } f \in S\left(L_{p}(\rho)\right) .\end{cases}$

Set

$$
C([0,1], \rho)=\left\{g / \rho^{1 / p}: g \in C[0,1]\right\}
$$

and

$$
W(\rho)=\left\{s / \rho^{1 / p}: s \in W\right\} .
$$

Then $C([0,1], \rho)$ is dense in $L_{p}([0,1], \rho)$ and $L_{p}([0,1], \rho)=\overline{W(\rho)}^{\|\cdot\|_{L_{p}(\rho)}}$. Moreover for a continuous function $g$, the set $A_{g}(\rho)=\left\{g_{a} / \rho^{1 / p}: a \in[1,2]\right\}$ is compact. Then the same arguments of Section 3 allow us to obtain the following. 
COROLlary 4.6. The mapping

$$
R: B\left(L_{p}(\rho)\right) \rightarrow S\left(L_{p}(\rho)\right)(1 \leqslant p<\infty)
$$

defined by $R f=Q_{\rho} f+P_{\rho} f$ is a 1-ball contractive retraction with $\omega(R)=1$.

In this section we have improved the results in the $L_{p}$ and $L_{\Phi}$ spaces of $[17,12]$, respectively. Though the mapping $Q$ is the same as the one introduced in those papers, here we construct in both cases a different retraction $R$ and, above all, our proofs are based on different ideas and techniques.

\section{The Lorentz Spaces $L^{p, q}$.}

Let $f^{*}$ denote the decreasing rearrangement of a function $f \in \mathcal{M}_{0}$, given by

$$
f^{*}(t)=\inf \{s \geqslant 0: \mu\{|f(x)|>s\} \leqslant t\}
$$

The Lorentz space $L^{p, q}:=L^{p, q}([0,1])(1 \leqslant q \leqslant p<\infty)$ consists of all $f \in \mathcal{M}_{0}$ for which the quantity

$$
\|f\|_{p, q}=\left(\frac{q}{p} \int_{[0,1]}\left(t^{1 / p} f^{*}(t)\right)^{q} \frac{d t}{t}\right)^{1 / q}
$$

is finite. As the Lorentz space $L^{p, q}$ is reflexive (see for example [14]) from [3, Corollary 4.4] it follows that it has absolutely continuous norm. Hence by Lemma 2.4 the space $L^{p, q}$ satisfies property (P1).

Lemma 5.1. Let $f \in L^{p, q}$ and $a \in[1,2]$, then

$$
\left(\frac{1}{a}\right)^{1 / p}\|f\|_{p, q}=\left\|f_{a}\right\|_{p, q} .
$$

Proof: Let $f \in L^{p, q}$. We observe that we have $\left(f_{a}\right)^{*}=\left(f^{*}\right)_{a}$. Then the lemma follows by a direct computation of $\left\|f_{a}\right\|_{p, q}^{q}$. Indeed we have

$$
\begin{aligned}
\left\|f_{a}\right\|_{p, q}^{q} & =\frac{q}{p} \int_{[0,1]} t^{(q / p)-1}\left(\left(f_{a}\right)^{*}(t)\right)^{q} d t=\frac{q}{p} \int_{[0,1]} t^{(q / p)-1}\left(\left(f^{*}\right)_{a}(t)\right)^{q} d t \\
& =\frac{q}{p} \int_{[0,1 / a]} t^{(q / p)-1}\left(f^{*}(a t)\right)^{q} d t \\
& =\left(\frac{1}{a}\right)^{q / p} \frac{q}{p} \int_{[0,1]} t^{(q / p)-1}\left(f^{*}(t)\right)^{q} d t=\left(\frac{1}{a}\right)^{(q / p)}\|f\|_{p, q}^{q},
\end{aligned}
$$

hence the thesis.

In view of Lemma 5.1 and Remark 3.7 we define $Q: B\left(L^{p, q}\right) \rightarrow B\left(L^{p, q}\right)$ (as in (7)) by

$$
(Q f)(t)=\left(\frac{2}{1+\|f\|_{p, q}}\right)^{1 / p} f_{2 /\left(1+\|f\|_{p, q}\right)}(t), \quad \text { for all } t \in[0,1] .
$$


Next define $P_{p, q}: B\left(L^{p, q}\right) \rightarrow L^{p, q}$

$$
P_{p, q} f= \begin{cases}\left(\frac{2}{1-\|f\|_{p, q}}\right)^{1 / p}\left(1-\|f\|_{p, q}^{q}\right)^{1 / q} \chi\left(\left(1+\|f\|_{p, q}\right) / 2,1\right] & \text { if } f \in B\left(L^{p, q}\right) \backslash S\left(L^{p, q}\right) \\ 0 & \text { if } f \in S\left(L^{p, q}\right) .\end{cases}
$$

We have that the mapping $P_{p, q}$ is compact and $\left\|Q f+P_{p, q} f\right\|_{p, q}=1$ for all $f \in B\left(L^{p, q}\right)$. Hence by Theorem 3.6 and Remark 3.7 we obtain the following.

TheOREM 5.2. The mapping

$$
R: B\left(L^{p, q}\right) \rightarrow S\left(L^{p, q}\right)(1 \leqslant q \leqslant p<\infty)
$$

defined by

$$
R f=Q f+P_{p, q} f
$$

is a 1-ball contractive retraction and $\omega(R)=1$.

The questions whether or not $W(X)=1$ in any infinite-dimensional Banach space $X$, and eventually if this value is always a minimum remain open.

We conclude this section with some remarks on fixed point free self-mappings of the unit ball $B(X)$. In [1, Theorem 3$]$ the following theorem has been proved.

THEOREM 5.3. Let $X$ be an infinite-dimensional Banach space and $\varepsilon>0$. Then there exists a fixed point free 1-ball contraction $F: B(X) \rightarrow B(X)$ with $\omega(F) \geqslant 1-\varepsilon$.

We have that, in some Banach spaces, the best value $\omega(F)=1$ can be attained by a fixed point free 1-ball contraction $F: B(X) \rightarrow B(X)$. Indeed if $R: B(X) \rightarrow S(X)$ is a $k$-ball contractive retraction, then $F=-R: B(X) \rightarrow B(X)$ is a fixed point free $k$-ball contraction. As a consequence of Corollary 4.6 and Theorem 5.2 we obtain the following.

COROLlary 5.4. Let $X$ denote either the weighted Lebesgue space $L_{p}(\rho)$ (1 $\leqslant p<\infty)$ or the Lorentz space $L^{p, q}(1 \leqslant q \leqslant p<\infty)$. Then there exists a fixed point free 1-ball contraction $F: B(X) \rightarrow B(X)$ with $\omega(F)=1$.

\section{BANACH SPACES WITH $(1+\varepsilon)$-BALL CONTRACTIVE RETRACTIONS.}

In this section we consider $X$ to be the space of all functions of absolutely continuous norm of a Banach function space $Y$, where $Y$ is either the grand $L^{p}$ space or the Marcinkiewicz spaces $M_{\beta}$. Applying Theorem 3.6 we prove that, in both cases, for any $\varepsilon>0$ there is a $(1+\varepsilon)$-ball contractive retraction $R$ with positive H-lower measure of noncompactness.

Let $1<p<\infty$. The grand $L^{p}$ space, which will be denoted by $L^{p)}:=L^{p)}([0,1])$, introduced in [11], is defined as the space of all functions $f \in \mathcal{M}_{0}$ such that

$$
\|f\|_{p)}=\sup _{0<\varepsilon<p-1}\left(\varepsilon \int_{[0,1]}|f(t)|^{p-\varepsilon} d t\right)^{1 /(p-\varepsilon)}<\infty .
$$


We denote by $X^{p)}$ the set of all functions in $L^{p)}$ of absolutely continuous norm and by $W$ the subset of $L^{p)}$ of all simple functions.

Lemma 6.1. The subspace $X^{p)}$ coincides with $\bar{W}^{\left(1 \cdot \|_{p}\right)}$, and the inclusion $X^{p)} \subset L^{p)}$ is proper.

Proof: Let $\sigma>0$ and set $\delta=(\sigma /(p-1))^{p}$. Let $D \in \Sigma$ with $\mu(D)<\delta$. As $\sup _{0<\varepsilon<p-1} \varepsilon^{1 /(p-\varepsilon)}=p-1$ and $\sup _{0<\varepsilon<p-1} \mu(D)^{1 /(p-\varepsilon)}=\mu(D)^{1 / p}$ we have

$$
\left\|\chi_{D}\right\|_{p)}=\sup _{0<\varepsilon<p-1}(\varepsilon \mu(D))^{1 /(p-\varepsilon)} \leqslant(p-1) \mu(D)^{1 / p}<\sigma .
$$

This shows that $\chi_{[0,1]}$ has absolutely continuous norm, hence by Lemma 2.4 (iii) it follows $X^{p)}=\bar{W}^{\left(\cdot \|_{p}\right)}$. To end the proof it suffices to note that the function $t^{-1 / p} \in L^{p)}$ has not absolutely continuous norm.

Lemma 6.2. Let $f \in X^{p)}$ and $a \in[1,2]$,

$$
\frac{1}{a}\|f\|_{p)} \leqslant\left\|f_{a}\right\|_{p)} \leqslant\|f\|_{p)} .
$$

Proof: For any $f \in X^{p)}$ and $a \in[1,2]$ we have

$$
\begin{aligned}
\left\|f_{a}\right\|_{p)} & =\sup _{0<\varepsilon<p-1}\left(\varepsilon \int_{\left[0, \frac{1}{a}\right]}|f(a t)|^{p-\varepsilon} d t\right)^{1 /(p-\varepsilon)} \\
& =\sup _{0<\varepsilon<p-1}\left(\frac{1}{a}\right)^{1 /(p-\varepsilon)}\left(\varepsilon \int_{[0,1]}|f(t)|^{p-\varepsilon} d t\right)^{1 /(p-\varepsilon)} \leqslant\|f\|_{p)} .
\end{aligned}
$$

On the other hand we find

$$
\|f\|_{p)}=\sup _{0<\varepsilon<p-1} a^{1 /(p-\varepsilon)}\left(\varepsilon \int_{[0,1 / a]}|f(a t)|^{p-\varepsilon} d t\right)^{1 /(p-\varepsilon)} \leqslant a\left\|f_{a}\right\|_{p)}
$$

which completes the proof.

Let $Q: B\left(X^{p)}\right) \rightarrow B\left(X^{p)}\right)$ be defined as in (3) and define for every $0<u<\infty$ the mapping $\left(P_{p)}\right)_{u}: B\left(X^{p)}\right) \rightarrow X^{p)}$ by

$$
\left(P_{p}\right)_{u} f= \begin{cases}\left.u \frac{1-\|Q f\|_{p)}}{\| \chi\left(\left(1+\|f\|_{p}\right)\right) / 2,1}\right] \|_{p)}^{\chi}\left(\left(1+\|f\|_{p}\right) / 2,1\right] & \text { if } f \in B\left(X^{p)}\right) \backslash S\left(X^{p)}\right) \\ 0 & \text { if } f \in S\left(X^{p)}\right) .\end{cases}
$$

Lемма 6.3. For any $0<u<\infty$, the mapping $\left(P_{p}\right)_{u}$ is compact, and for $f \in B\left(X^{p)}\right)$

$$
\left\|\left(P_{p)}\right)_{u} f\right\|_{p)}=u\left(1-\|Q f\|_{p)}\right)
$$

Proof: The proof that $\left(P_{p)}\right)_{u}$ is compact is similar to the proof of Lemma 4.2. A direct calculation gives the norm of $\left(P_{p}\right)_{u} f$. 
Lemma 6.4. Let $0<u<\infty$. For any $f \in B\left(X^{p)}\right)$

$$
\max \{1, u\} \geqslant\left\|Q f+\left(P_{p)}\right)_{u} f\right\|_{p)} \geqslant \frac{u}{u+1}
$$

Proof: Let $f \in B\left(X^{p)}\right)$, then

$$
\left\|Q f+\left(P_{p)}\right)_{u} f\right\|_{p)}=\sup _{0<\varepsilon<p-1}\left(\varepsilon \int_{[0,1]}|(Q f)(t)|^{p-\varepsilon} d t+\varepsilon \int_{[0,1]}\left|\left(\left(P_{p)}\right)_{u} f\right)(t)\right|^{p-\varepsilon} d t\right)^{1 /(p-\varepsilon)} .
$$

Now for any fixed $0<\varepsilon<p-1$

$$
\varepsilon \int_{[0,1]}|(Q f)(t)|^{p-\varepsilon} d t \leqslant \varepsilon \int_{[0,1]}|(Q f)(t)|^{p-\varepsilon} d t+\varepsilon \int_{[0,1]}\left|\left(\left(P_{p)}\right)_{u} f\right)(t)\right|^{p-\varepsilon} d t
$$

and passing to the $1 /(p-\varepsilon)$-power we have

$$
\left(\varepsilon \int_{[0,1]}|(Q f)(t)|^{p-\varepsilon} d t\right)^{1 /(p-\varepsilon)} \leqslant\left(\varepsilon \int_{[0,1]}|(Q f)(t)|^{p-\varepsilon} d t+\varepsilon \int_{[0,1]}\left|\left(\left(P_{p)}\right)_{u} f\right)(t)\right|^{p-\varepsilon} d t\right)^{1 /(p-\varepsilon)}
$$

Taking the supremum over $\varepsilon$ we get $\left\|Q f+\left(P_{p)}\right)_{u} f\right\|_{p)} \geqslant\|Q f\|_{p)}$. Analogously we get

$$
\left\|Q f+\left(P_{p)}\right)_{u} f\right\|_{p)} \geqslant\left\|\left(P_{p)}\right)_{u} f\right\|_{p)}
$$

Then

$$
\left\|Q f+\left(P_{p)}\right)_{u} f\right\|_{p)} \geqslant \max \left\{\|Q f\|_{p)}, u\left(1-\|Q f\|_{p)}\right)\right\} \geqslant u / u+1
$$

On the other hand it easily follows

$$
\left\|Q f+\left(P_{p)}\right)_{u} f\right\|_{p)} \leqslant\|Q f\|_{p)}+u\left(1-\|Q f\|_{p)}\right) \leqslant \max \{1, u\} .
$$

By Lemmas 6.1 and 6.2, the Banach space $X^{p)}$ satisfies properties (P1) and (P2). Hence by Lemmas 6.3 and 6.4 and Theorem 3.6 we have that the mapping. $R_{u}: B\left(X_{p)}\right)$ $\rightarrow S\left(X_{p)}\right)$ defined by

$$
R_{u} f=\frac{Q f+\left(P_{p)}\right)_{u} f}{\left\|Q f+\left(P_{p)}\right)_{u} f\right\|_{p)}}
$$

is $(u+1) / u$-ball contractive with $\omega\left(R_{u}\right) \geqslant \min \{1 / 2,1 /(2 u)\}$. As $\lim _{u \rightarrow \infty}(u+1) / u=1$ we obtain the following theorem.

THEOREM 6.5. For any $\varepsilon>0$ there is a retraction

$$
R: B\left(X^{p)}\right) \rightarrow S\left(X^{p)}\right)(1<p<\infty)
$$

which is $(1+\varepsilon)$-ball contractive with $\omega(R)>0$. 
REMARK 6.6. The same result of Theorem 6.5 can be proved in the small Lebesgue space $L^{p)^{\prime}}(1<p<\infty)$ introduced in [7], in which the norm is defined as

$$
\|f\|_{p)^{\prime}}=\sup _{g \in L^{p)}} \frac{\int_{[0,1]} f(t) g(t) d t}{\|g\|_{p)}} .
$$

We recall that the spaces $L^{p)^{\prime}}$ have absolutely continuous norm, and the spaces $L^{p)}$ are characterised as dual spaces of $L^{p)^{\prime}}$ (see [5]).

An analogous result holds in the Marcinkiewicz space

$$
M_{\beta}:=M_{\beta}([0,1])(0<\beta<1)
$$

which consists of all $f \in \mathcal{M}_{0}$ for which

$$
\|f\|_{\beta}=\sup \frac{1}{\mu(E)^{\beta}} \int_{E}|f(t)| d t<\infty .
$$

where the supremum is taken over all $E \in \Sigma$ with $\mu(E)>0$. We denote by $X_{\beta}$ the set of all functions in $M_{\beta}$ of absolutely continuous norm and $W$ the subset of $M_{\beta}$ of all simple functions.

LEMMA 6.7 . The subspace $X_{\beta}$ coincides with $\bar{W}^{\|\cdot\|_{\beta}}$, and the inclusion $X_{\beta} \subset M_{\beta}$ is proper.

Proof: We prove that for every $D \in \Sigma$

$$
\left\|\chi_{D}\right\|_{\beta}=\mu(D)^{1-\beta}
$$

By definition we have

$$
\left\|\chi_{D}\right\|_{\beta}=\sup \frac{1}{\mu(E)^{\beta}} \mu(D \cap E) .
$$

Choose for every $n \in N$ a set $E_{n} \in \Sigma$ such that

$$
\left\|\chi_{D}\right\|_{\beta}-\frac{1}{n} \leqslant \frac{1}{\mu\left(E_{n}\right)^{\beta}} \mu\left(D \cap E_{n}\right) \leqslant\left\|\chi_{D}\right\|_{\beta} .
$$

Set $D_{n}=D \cap E_{n}$. As $D_{n} \subset E_{n}$ we get $1 /\left(\mu\left(E_{n}\right)^{\beta}\right) \leqslant 1 /\left(\mu\left(D_{n}\right)^{\beta}\right)$. Consequently,

$$
\left\|\chi_{D}\right\|_{\beta}-\frac{1}{n} \leqslant \frac{1}{\mu\left(E_{n}\right)^{\beta}} \mu\left(D_{n}\right) \leqslant \frac{1}{\mu\left(D_{n}\right)^{\beta}} \mu\left(D_{n}\right) \leqslant\left\|\chi_{D}\right\|_{\beta} .
$$

As $n$ goes to infinity we get (8). From (8) it obviously follows that $\chi_{[0,1]}$ has absolutely continuous norm, hence (iii) of Lemma 2.4 gives $X_{\beta}=\bar{W}^{\|\cdot\|_{s}}$. As pointed out in [2] the space $M_{\beta}$ has not absolutely continuous norm.

It easy to check that the following lemma holds. 
Lemma 6.8. Let $f \in X_{\beta}$ and $a \in[1,2]$,

$$
\left(\frac{1}{a}\right)^{1-\beta}\|f\|_{\beta} \leqslant\left\|f_{a}\right\|_{\beta} \leqslant\|f\|_{\beta} .
$$

Now let $Q: B\left(X_{\beta}\right) \rightarrow B\left(X_{\beta}\right)$ be defined as in (3) and define for every $0<u<\infty$ the mapping $\left(P_{\beta}\right)_{u}: B\left(X_{\beta}\right) \rightarrow X_{\beta}$ by

$$
\left(P_{\beta}\right)_{u} f= \begin{cases}u\left(\frac{2}{1-\|f\|_{\beta}}\right)^{1-\beta}\left(1-\|Q f\|_{\beta}\right) \chi\left(\left(1+\|f\|_{\beta}\right) / 2,1\right] & \text { if } f \in B\left(X_{\beta}\right) \backslash S\left(X_{\beta}\right) \\ 0 & \text { if } f \in S\left(X_{\beta}\right) .\end{cases}
$$

For every $0<u<\infty$, the mapping $\left(P_{\beta}\right)_{u}$ is compact and

$$
\left\|\left(P_{\beta}\right)_{u} f\right\|_{\beta}=u\left(1-\|Q f\|_{\beta}\right) .
$$

Moreover the following estimates of $\left\|Q f+\left(P_{\beta}\right)_{u} f\right\|_{\beta}$ can be derived by an argument similar to that of Lemma 6.4.

Lemma 6.9. Let $0<u<\infty$. For any $f \in B\left(X_{\beta}\right)$

$$
\max \{1, u\} \geqslant\left\|Q f+\left(P_{\beta}\right)_{u} f\right\|_{\beta} \geqslant \frac{u}{u+1} .
$$

By Lemmas 6.7 and 6.8, the Banach space $X_{\beta}$ satisfies properties (P1) and (P2). Then by the previous Lemma and Theorem 3.6 we have that the mapping $R_{u}: B\left(X_{\beta}\right)$ $\rightarrow S\left(X_{\beta}\right)$ defined by

$$
R_{u} f=\frac{Q f+\left(P_{\beta}\right)_{u} f}{\left\|Q f+\left(P_{\beta}\right)_{u} f\right\|_{\beta}}
$$

is $(u+1) / u$-ball contractive with

$$
\omega\left(R_{u}\right) \geqslant \min \left\{1 /\left(2^{1-\beta} u\right), 1 /\left(2^{1-\beta}\right)\right\} .
$$

As $\lim _{u \rightarrow \infty}(u+1) / u=1$ we obtain the following.

THEOREM 6.10. For any $\varepsilon>0$ there is a retraction $R: B\left(X_{\beta}\right) \rightarrow S\left(X_{\beta}\right)$ which is $(1+\varepsilon)$-ball contractive with $\omega(R)>0$.

\section{REFERENCES}

[1] J. Appell, N.A. Erkazova, S. Falcon Santana and M. Väth, 'On some Banach space constants arising in nonlinear fixed point and eigenvalue theory', Fixed Point Theory Appl. 4 (2004), 317-336.

[2] J. Appell and P.P. Zabrejko, Nonlinear superposition operators (Cambridge University Press, Cambridge, 1990).

[3] C. Bennett and R. Sharpley, Interpolation of operators, Pure and Applied Maths 129 (Boston Academic Press, Boston MA, 1988). 
[4] Y. Benyamini and Y. Sternfeld, 'Spheres in infinite-dimensional normed spaces are Lipschitz contractible', Proc. Amer. Math. Soc. 88 (1983), 439-445.

[5] C. Capone and A. Fiorenza, 'On small Lebesgue spaces', J. Funct. Spaces Appl. 3 (2005), 73-89.

[6] D. Caponetti and G. Trombetta, 'On proper $k$-ball contractive retractions in the Banach space $B C([0, \infty))$ ', Nonlinear Func. Anal. Appl. (to appear).

[7] A. Fiorenza, 'Duality and reflexivity in grand Lebesgue spaces', Collect. Math. 51 (2000), 131-148.

[8] K. Goebel, 'On the minimal displacement of points under Lipschitzian mappings', Pacific J. Math. 45 (1973), 151-163.

[9] K. Goebel, 'On the problem of retracting balls onto their boundary', Abstr. Appl. Anal. 2 (2003), 101-110.

[10] K. Goebel and W.A. Kirk, Topics in metric fixed point theory (Cambridge, 1990).

[11] T. Iwaniec and C. Sbordone, 'On the integrability of the Jacobian under minimal hypotheses', Arch. Rational Mech. Anal. 119 (1992), 129-143.

[12] G. Lewicki and G. Trombetta, 'Almost contractive retractions in Orlicz spaces', Bull. Austral. Math. Soc. 68 (2003), 353-369.

[13] B. Nowak, 'On the Lipschitzian retraction of the unit ball in infinite-dimensional Banach spaces onto its boundary', Bull. Acad. Polon. Sci. 27 (1979), 861-864.

[14] M.M. Rao and Z.D. Ren, Theory of Orlicz spaces, Monographs and Textbooks in Pure and Applied Mathematics 146 (Marcel Dekker, Inc., New York, 1991).

[15] W. Rudin, Real and complex analysis (McGraw-Hill Book Co., New York, 1987).

[16] G. Trombetta, ' $k$-set contractive retractions in spaces of continuous functions', Sci. Math. Jpn. 59 (2004), 121-128.

[17] A. Trombetta and G. Trombetta, 'On the existence of $\left(\gamma_{p}\right)$ k-set contractive retractions in $L_{p}[0,1]$ spaces, $1 \leqslant p<\infty$ ', Sci. Math. Jpn. 56 (2002), 327-335.

[18] M. Väth, Ideal spaces, Lect. Notes in Math. 1664 (Springer-Verlag, Berlin, 1997).

[19] M. Väth, 'On the minimal displacement problem of $\gamma$-Lipschitz maps and $\gamma$-Lipschitz retractions onto the sphere', Z. Anal. Anwendungen 21 (2002), 901-914.

[20] J. Wośko, 'An example related to the retraction problem', Ann. Univ. Mariae Curie-Sktodowska 45 (1991), 127-130.

Department of Mathematics

University of Palermo

Via Archirafi, 34

90123 Palermo

Italy

e-mail: d.caponetti@math.unipa.it
Department of Mathematics

University of Calabria

87036 Arcavacata di Rende (CS)

Italy

e-mail: aletromb@unical.it

Department of Mathematics

University of Calabria

87036 Arcavacata di Rende (CS)

Italy

e-mail: trombetta@unical.it 